\title{
Improving the Predictive Capability of Popular SWCCs by Incorporating Maximum Possible Suction
}

\author{
Shada H. Krishnapillai, Nadarajah Ravichandran \\ Civil Engineering Department, Clemson University, Clemson, USA \\ E-mail:nravic@clemson.edu \\ Received July 12, 2011; revised August 27, 2011; accepted October 6, 2011
}

\begin{abstract}
Soil-water characteristic curve (SWCC) that represents the relationship between the soil moisture and matric suction is one of the important constitutive models required for numerical modeling of unsaturated soils. An effective SWCC model should be capable of calculating the moisture-suction variation for the entire range of degree of saturation. Applicability of popular SWCC models such as Brooks and Corey, van Genuchten, and Fredlund and Xing is limited, especially in low $(<20 \%)$ degree of saturation range. In this study, all these models are modified by incorporating maximum suction as one of the model parameters, so that these models can be effectively used over the entire range of degree of saturation. The Fredlund et al. (1994) permeability function is also modified based on the modification to the Fredlund and Xing SWCC model. The applicability of the improved models is investigated by calibrating the SWCC of various types of soil and presented in this paper. Based on this study it can be concluded that the modified models are flexible enough to fit the experimental data for the entire range of degree of saturation.
\end{abstract}

Keywords: Unsaturated Soils, Soil Water Characteristic Curve, Permeability Function, Relative Permeability of Unsaturated Soils, Relative Permeability Using Soil Water Characteristic Curve

\section{Introduction}

Unsaturated soil is a three phase porous media consisting of three bulk phases: solid skeleton, water, and pore air. In addition to these three bulk phases, there exist three interfaces: solid-water interface, solid-air interface, and water-air interface. Of the three interfaces, the water-air interface also known as contractile skin that does not exist in either saturated or dry soil influences the flow and mechanical behavior of unsaturated soil. The contractile skin maintains the pressure balance between water and air phases. The difference between the air pressure and water pressure is known as matric suction.

\subsection{Soil Water Characteristic Curves}

The Soil Water Characteristic Curves (SWCC) is a relationship between the amount of water present in the soil (moisture) and the suction characteristics of the soil matrix. The amount of water present in the soil can be expressed in terms of degree of saturation $(S)$, volumetric water content $(\theta)$, or gravimetric water content $(w)$. Many researchers have identified the factors which in- fluence the shape of the SWCC and based on that, many mathematical SWCC models were developed. These basic soil properties include void ratio, void distribution, particle size distribution and initial density. Gardner [1], Brooks and Corey [2], van Genuchten [3], Kosugi [4], and Fredlund and Xing [5] are some of the notable mathematical models found in the literature. All these models confirm an inverse proportional relationship between $S$ and suction $(\psi)$. This can be explained with the fundamental meniscus theory shown in Equation (1). When the $S$ increases, the radius of the meniscus $\left(R_{s}\right)$ will increase. When $R_{s}$ increases, the pressure difference between the pore air pressure and the pore water pressure (matric suction) will decrease.

$$
\psi=\left(p^{g}-p^{l}\right)=\frac{2 T_{s}}{R_{s}}
$$

where $\psi_{m}$ is the suction, $p^{g}$ is pore gas pressure, $p^{l}$ is pore liquid pressure, and $T_{s}$ is surface tension.

The air-entry value and pore size distribution are two basic parameters incorporated in widely used Brooks and Corey $(B-C)$, van Genuchten $(v-G)$, and Fredlund and 
Xing $(F-X)$ and are denoted by $a$ and $n$, respectively.

The $B-C$ model shown in Equation (2) is one of the basic SWCC models developed with two parameters. Although this is widely used model, it does not provide a continuous mathematical function for the entire range of S.

$$
\frac{\theta-\theta_{r}}{\theta_{s}-\theta_{r}}=\frac{1}{\left(\ln \left(e+(\psi / a)^{n}\right)\right)^{m}}
$$

where $a$ and $n$ are the fitting parameters. The parameter $a$ is related to the air-entry suction of the soil and the $n$ is related to the pore size distribution of the soil, $\theta$ is volumetric water content, $\theta_{r}$ is residual water content, and $\theta_{s}$ is saturated water content.

The $v$ - $G$ model shown in Equation (3) provides a single equation for the entire range of $\mathrm{S}$. This model has an additional fitting parameter $m$, thereby making this model more flexible compared to the B-C model.

$$
\frac{\theta-\theta_{r}}{\theta_{s}-\theta_{r}}=\frac{1}{\left(1+(a \psi)^{n}\right)^{m}}
$$

where the fitting parameter $m$ is related to residual water content. In some versions the parameter $m$ is related to $n$ resulting in only two independent parameters $a$ and $n$. Other parameters are same as in the $B-C$ model.

The $F$ - $X$ model is shown in Equation (4). The $F$ - $X$ model assumes a maximum suction of $1,000,000 \mathrm{kPa}$ at dry condition, while the $B-C$ and the $v-G$ models assume infinite value of maximum suction. This maximum possible suction of $1,000,000 \mathrm{kPa}$ for any soil is based on thermodynamic principles rather than actual measurement. The $F-X$ model is similar to the $\mathrm{v}-\mathrm{G}$ model other than the correction factor $C(\psi)$ and the logarithmic function in the equation. The logarithmic function is the key in the $F$ - $X$ equation (Equation (4)). It keeps the denominator a nonzero value for any suction value. The corresponding correction function is shown in Equation (5). This equation can be literally used for degree of saturation that is below residual value. However, Fredlund and Xing [5] suggested another form of the same model which is shown in Equation (5). This second form can be used if a residual water content is known.

$$
\begin{gathered}
\frac{\theta}{\theta_{s}}=\frac{C(\psi)}{\left(\ln \left(e+(\psi / a)^{n}\right)\right)^{m}} \\
C(\psi)=1-\frac{\ln \left(1+\psi / \psi_{r}\right)}{\ln \left(1+10^{6} / \psi_{r}\right)} \\
\frac{\theta-\theta_{r}}{\theta_{s}-\theta_{r}}=\frac{1}{\left(\ln \left(e+(\psi / a)^{n}\right)\right)^{m}}
\end{gathered}
$$

where $\psi_{r}$ is the suction corresponding to the residual water content $\theta_{r}$ and other parameters are same as in the $v-G$ model.

\subsection{Relative Permeability Functions}

Accurate evaluation of the permeability is important for accurate modeling of flow and deformation problems in unsaturated soils. Because the permeability of unsaturated soil is uniquely influenced by the degree of saturation [6], the soil water characteristic curve (SWCC) of the soil can be used to predict the permeability coefficient. The SWCC is a unique constitutive equation in unsaturated soil that relates the degree of saturation to the matric suction and it incorporates the basic soil properties associated with flow such as void ratio, pore size distribution, void distribution, particle size distribution and initial density. The major advantage of using the SWCC is that the moisture-suction relationship can be easily obtained experimentally than the moisture-permeability relationship.

Based on $F-X$ SWCC model, the permeability function shown in Equation (7) was proposed by Fredlund et al. [6].

$$
K_{r}(\psi)=\frac{\int_{\ln (\psi)}^{b} \frac{\theta\left(e^{y}\right)-\theta(\psi)}{e^{y}} \theta^{\prime}\left(e^{y}\right) \mathrm{d} y}{\int_{\ln \left(\psi_{\text {aev }}\right)}^{b} \frac{\theta\left(e^{y}\right)-\theta_{s}}{e^{y}} \theta^{\prime}\left(e^{y}\right) \mathrm{d} y}
$$

where $\psi$ is suction, $K_{r}(\psi)$ is the relative permeability at suction $\psi, \psi_{a e v}$ is the air-entry suction, $\mathrm{y}$ is a dummy variable of integration, $b=\ln (1,000,000), \theta$ is volumetric water content given in Equation (4) and $\theta^{\prime}$ is its derivative. $a, n, m$ and $C_{r}$ are fitting parameters of the $F-X$ model (Equation (4)).

\subsection{Need for Modification to the Existing Popular SWCC and Relative Permeability Model}

The $B-C, v-G$, and $F-X$ models are being widely used to calculate the moisture-suction relation of unsaturated soils. For the $B-C$ and $v-G$ models, a residual water content value has to be specified. However these two models calculate unrealistic suction when the normalized water content is zero or less, i.e. water content of the soil is less than or equal to the residual water content. In the $F-X$ model, the maximum suction is assumed to be $1,000,000$ $\mathrm{kPa}$. Although there are thermodynamic concepts to back up this maximum suction, it is a concern to use a fixed value for all types of soils. In addition, when the actual 
maximum suction is low, usage of such larger maximum suction value might over predict shear strength in numerical simulations. Similar to the $B-C$ and $v-G$ models, the second form of the $F-X$ model (Equation (5)) also calculates an unrealistic suction when the normalized water content is zero or less. Therefore, to avoid an unrealistic suction value at zero normalized water content, the maximum suction value should be specified even with a residual water content specified. In addition, the fourth model parameter $C_{r}$ in the $F-X$ model is chosen from a wide range ( 1 to $1,000,000 \mathrm{kPa}$ ) and it creates difficulties in achieving a unique set of calibrated model parameters. Also, the $C_{r}$ affects the initial portion of the curve when the value of $C_{r}$ is relatively low and it is considered as another disadvantage [6]. The primary objective of this study is to increase the flexibility of the $B-C$ and $v-G$ models so that these models can predict realistic high suctions in low degree of saturations without causing numerical instabilities in finite element simulations. That is, in the modeling the dynamic behavior of unsaturated soils, there are numerous constitutive relations that can cause "numerical problems" during the simulation. The best example is the nonlinear constitutive model (elastoplastic stress-strain model). The other one is the soil water characteristic curve which is used to calculate the suction value at a given time increment for the calculated degree of saturation or vise versa. If the calculated suction is really large or really small, it can introduce really large or really small number in one of the element matrices (mass, damping or fluid stiffness). This might result in crashing the program even with really small time increment.

It is very challenging to model the soil behavior from a fully dry condition to a fully saturated condition using a single fully coupled finite element computer code. The current state of the art suggests that there are three major difficulties in developing numerically stable simulation capability. They are: difficulties in dealing with multiple nodal/element variables in finite element formulation of porous media at these extreme conditions, difficulties in developing stress-strain behavior with appropriate stress state variables at these extreme conditions, and difficulties in accurately calculating the suction over the entire range of degree of saturation. The modified models can be incorporated in finite element simulation without introducing numerical instabilities from SWCC. The idea here is to modify the original curve in such a way it calculates a finite number at very low degree of saturation. In the original model, the curve is very steep at low degree of saturation. A small change in degree of saturation can result in unrealistic suction value at very low degree of saturation. Since the modified curve calculates finite values, numerical instability can be reduced or elimi- nated.

In this study, the $B-C$ and $v-G$ models are modified by incorporating correction factors. Also, the correction factor in the $F-X$ model is modified to avoid the effects of additional fitting parameter $C_{r}$. Incorporating the maximum suction as part of the model increased its flexibility in fitting measured data of various soils over the full range of $S$. All three models are improved with the feature to specify both residual water content and maximum suction values. The capability of the improved models is verified by matching with the experimental data and prediction of original models. Based on the improved $F-X$ model, the permeability function proposed by Fredlund et al. [6] is modified and presented.

\section{Improved SWCC Models and Comparisons}

Although there are numerous SWCC models available in the literature, this study is intended to improve the popular $B-C, v-G$, and $F-X$ models. The $B-C$ and $v-G$ models are modified primarily to make sure that these models no longer calculate high suction when the normalized water content is zero or less. And also the modified models have the feature to specify both residual water content and maximum suction values.

\subsection{The Improved Brooks and Corey $(I-B-C)$ Model}

The improved Brooks and Corey $(I-B-C)$ model is given in Equation (8). To preserve the advantage of the $B-C$ model, no additional fitting parameter is introduced. Even though the maximum suction $\psi_{\max }$ is incorporated in the equation, it cannot be considered to be a fitting parameter, as the shape of the SWCC cannot be changed by adjusting the $\psi_{\max }$. The $I-B-C$ model does not provide a continuous mathematical function for the entire range of degree of saturation but gives a finite number of suction value at very low degree of saturation value.

$$
\frac{\theta-\theta_{r}}{\theta_{s}-\theta_{r}}=\left\{\begin{array}{cc}
1 & \text { if } \psi<a \\
\frac{C(\psi)}{(\psi / a)^{n}} & \text { if } \psi>a
\end{array}\right.
$$

The correction function for the $I-B-C$ model is shown below in Equation (9). A trial and error procedure was followed to fit the data and there was no theoretical basis for the equation for the correction function. An obvious condition needed to be satisfied was to obtain the correction function but to obtain $C(\psi)$ is equal to zero at the residual water content.

$$
C(\psi)=1-\sqrt{\psi / \psi_{r}}
$$


where $\psi_{r}$ is suction at residual water content $\theta_{r}$ and other parameters are same as in the $B$ - $C$ model. If the residual water content is set to zero, then $\psi_{r}$ becomes $\psi_{\text {max }}$.

\subsection{Comparison of the $B-C$ and the $I-B-C$ Models}

The capability of the Improved $B-C(I-B-C)$ model in predicting the moisture-suction relation is investigated and compared with the $B-C$ model for four different soils. The comparison of $B-C$ and $I-B-C$ Models for Columbia sandy loam (data from [2]) is shown in Figure 1. The Figures 2 and 3 show the comparison for Madrid clay sand and Arlington soil, respectively. The Figures $\mathbf{4}$ shows the comparison for Indian head till (data from [8]).

It should be noted that the experimental SWCC data are not available for the full range of $S(0 \%-100 \%)$. Based on the experimental data, the maximum suction of $1,000,000 \mathrm{kPa}$ is chosen for all four soils. The residual water content is assumed to be zero for all four soils. As shown in these figures, the predictions by $I-B-C$ model shows slight improvement compared to the original $B-C$ model. The $B-C, I-B-C$ models are not effective for

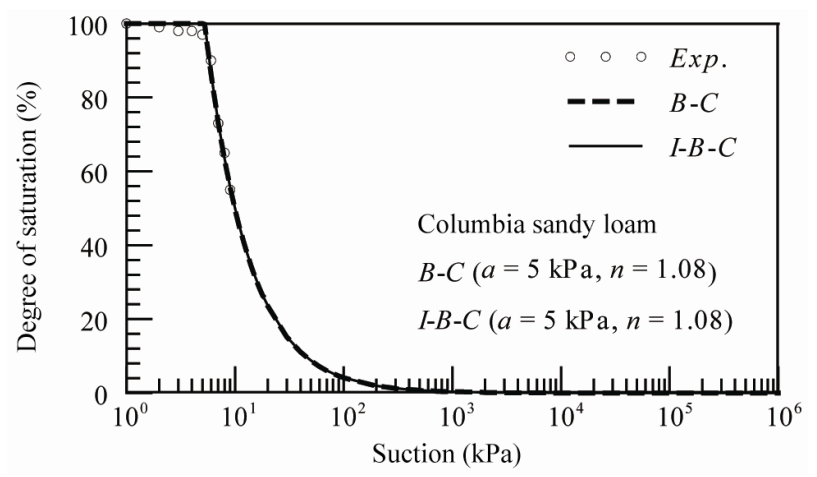

Figure 1. B-C and $I-B-C$ SWCCs for Columbia sandy loam.

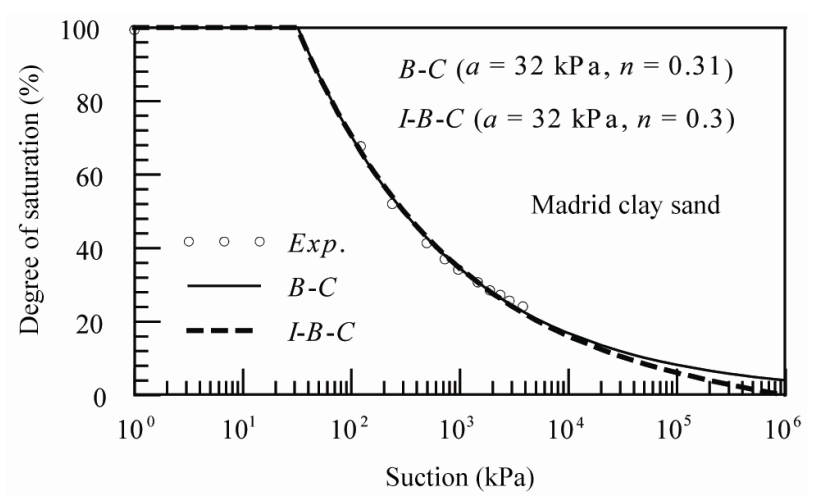

Figure 2. B-C and I-B-C SWCCs for Madrid clay sand.

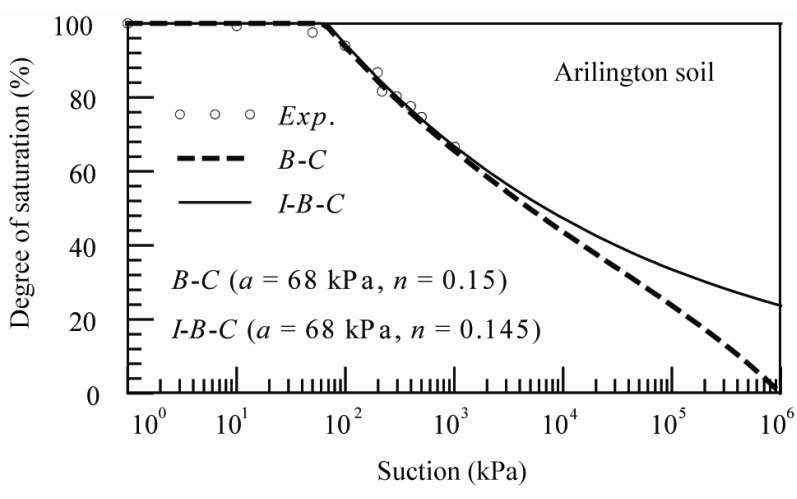

Figure 3. B-C and I-B-C SWCCs for Arlington soil.

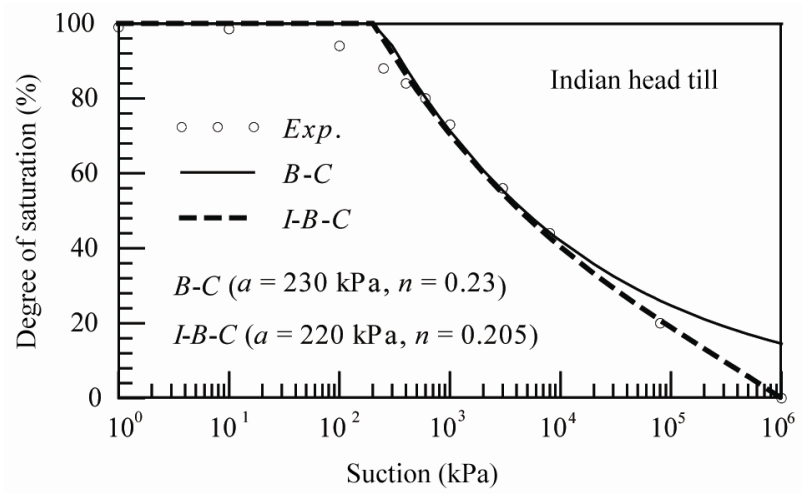

Figure 4. B-C and I-B-C SWCCs for Indian head till.

sandy soils and it is evidently shown in Figure $\mathbf{1}$ as these models failed to keep the shape of the SWCC without reaching zero normalized water content in low suction range.

\subsection{The Improved van Genuchten (I-v-G) Model}

The Improved van Genuchten $(I-v-G)$ model is given in Equation (10). Since the parameter $a$ is related to the airentry suction, the model is revised so that the parameter $a$ has the unit of suction. The $I-v-G$ model is developed with the feature to specify both residual water content and maximum suction value with no additional fitting parameter.

$$
\frac{\theta-\theta_{r}}{\theta_{s}-\theta_{r}}=\frac{C(\psi)}{\left(1+(\psi / a)^{n}\right)^{m}}
$$

The modified correction function is shown below in Equation (11).

$$
C(\psi)=1-\left(\frac{m+1}{m+\left(\psi_{\max } / \psi\right)}\right)^{0.5}
$$

where $\psi_{\max }$ is maximum suction and other parameters are 
same as in the $v$ - $G$ model.

\subsection{Predictive Capability of the $I-v-G$ Model}

Capability of the Improved $v-G(I-v-G)$ model in predicting the moisture-suction relation is presented for Columbia sandy loam, Madrid clay sand, Arlington soil, and Indian head till in Figures 5-8, respectively. Similar to the $I-B-C$ model, maximum suction of $1,000,000 \mathrm{kPa}$ and residual water content of zero are used for all four soils. As shown in Figures 5-8, the $I-v-G$ model is capa-

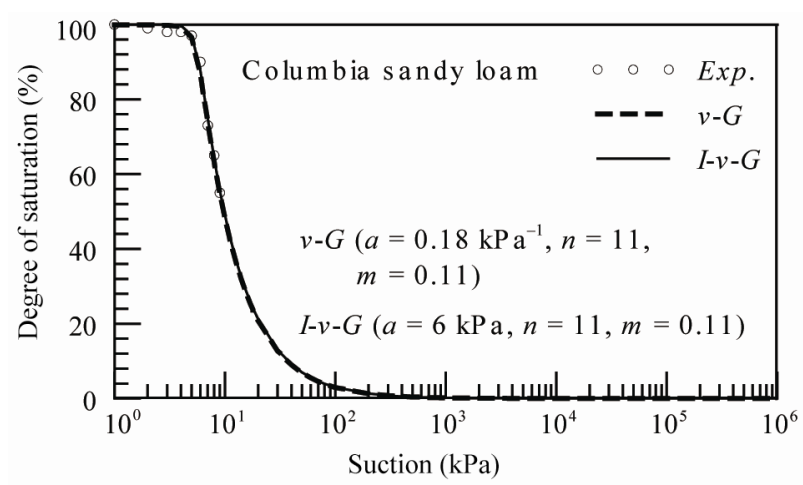

Figure 5. v-G and $I-v-G$ SWCCs for Columbia sandy loam.

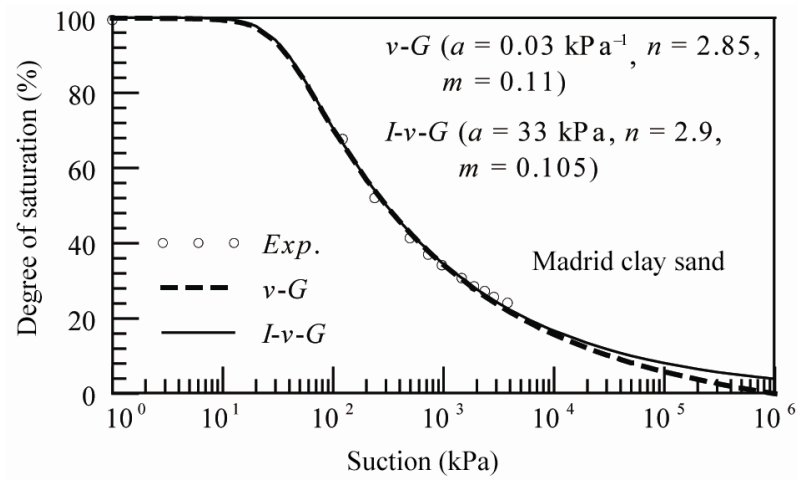

Figure 6. $v-G$ and $I-v-G$ SWCCs for Madrid clay sand.

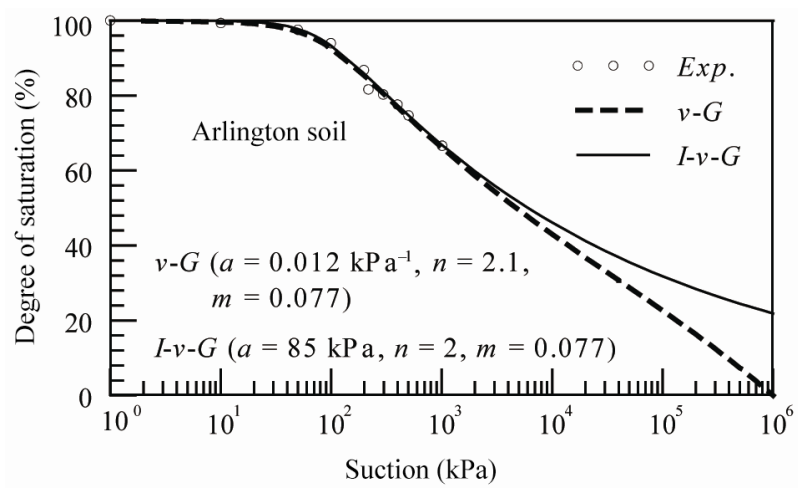

Figure 7. $v-G$ and $I-v-G$ SWCCs for Arlington soil.

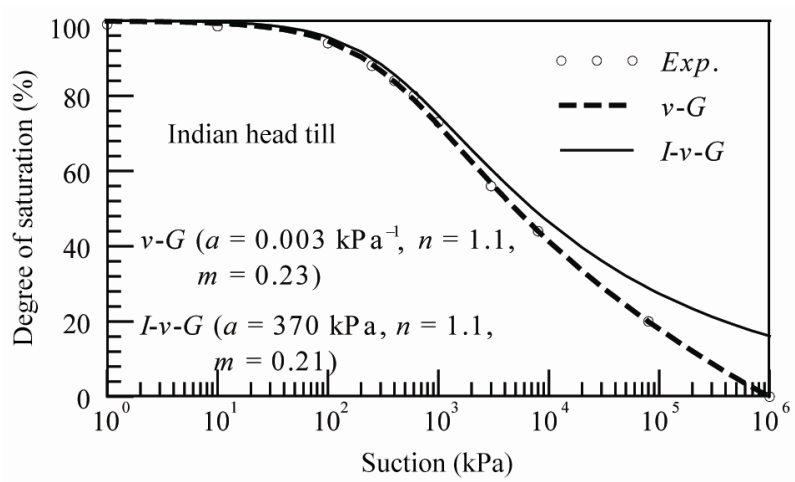

Figure 8. $v-G$ and $I-v-G$ SWCCs for Indian head till.

ble of calculating the moisture-suction relation for full range of $\mathrm{S}$, whereas the $v$ - $G$ model is not effective. As shown in Figure 5, the $v-G, I-v-G$ models are also not suitable for sandy soils as these models also failed to keep the SWCC without reaching zero normalized water content in low suction range.

\subsection{The Improved Fredlund and Xing $(I-F-X)$ Model}

The Improved Fredlund and Xing $(I-F-X)$ model is given in Equation (12). The $I-F-X$ model is developed with the feature to specify both residual water content and maximum suction value without the parameter $C_{r}$, i.e. with only three fitting parameters. Therefore, the effect of $C_{r}$ in the initial portion of the $F-X$ model [7] is avoided in the $I-F-X$ model.

$$
\frac{\theta-\theta_{r}}{\theta_{s}-\theta_{r}}=\frac{C(\psi)}{\left(\ln \left(e+(\psi / a)^{n}\right)\right)^{m}}
$$

The modified correction function is shown below in Equation (13).

$$
C(\psi)=1-\left(\frac{m+1}{m+\left(\psi_{\max } / \psi\right)}\right)^{0.5}
$$

where all the parameters are same as in the $I-v-G$ model.

\subsection{Predictive Capability of the $I-F-X$ Model}

The predictive capability of the $I-F-X$ model in predicting the moisture-suction relation is presented in Figures 9-12. Similar to the $I-B-C, I-v-G$ models, $1,000,000 \mathrm{kPa}$ maximum suction and zero residual water content are used. It can be noted that the $I-F-X$ model is also effective in full range of $\mathrm{S}$. However the $I-F-X$ model can be considered better as it has only three fitting parameters, whereas the $F$ - $X$ model has four. 


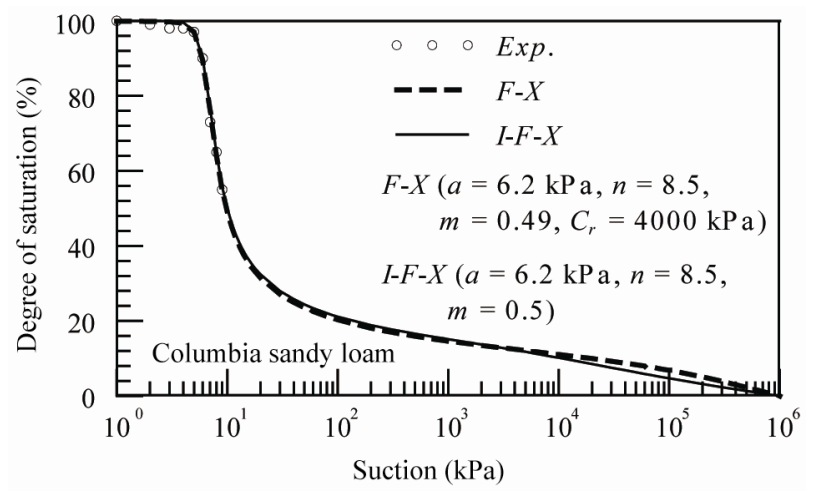

Figure 9. F-X and I-F-X SWCCs for Columbia sandy loam.

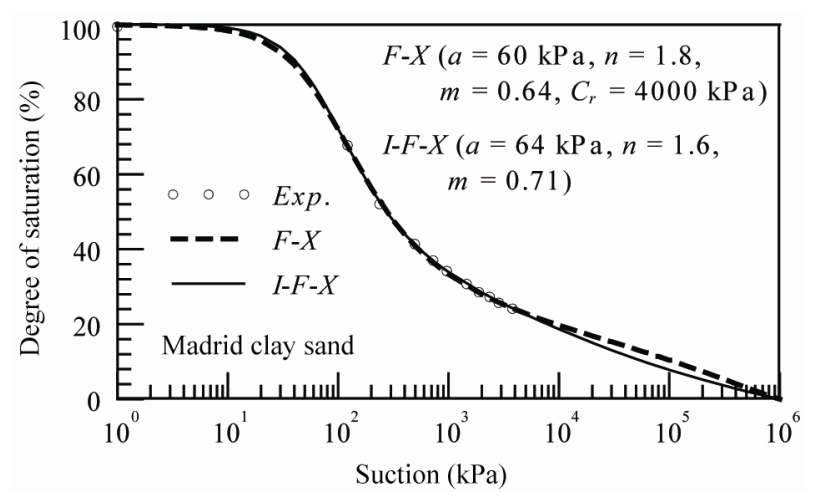

Figure 10. F-X and I-F-X SWCCs for Madrid clay sand.

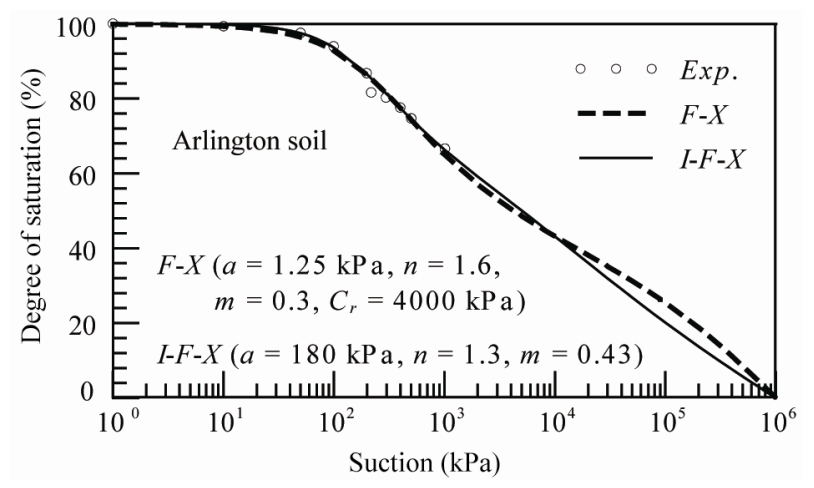

Figure 11. F-X and I-F-X SWCCs for Arlington soil.

\section{Modified Permeability Function and Comparisons}

Based on $F$ - $X$ SWCC model, a permeability function is proposed by Fredlund et al. [7] and has been widely used. Therefore, it is important to modify the Fredlund et al permeability function ( $F$-All model) based on the $I-F-X$ SWCC model. The $F$-All model is modified based on the $I-F-X$ SWCC model, and presented as $I-F$-All model in Equation (14). One can immediately see that the improved and original relative permeability equations are

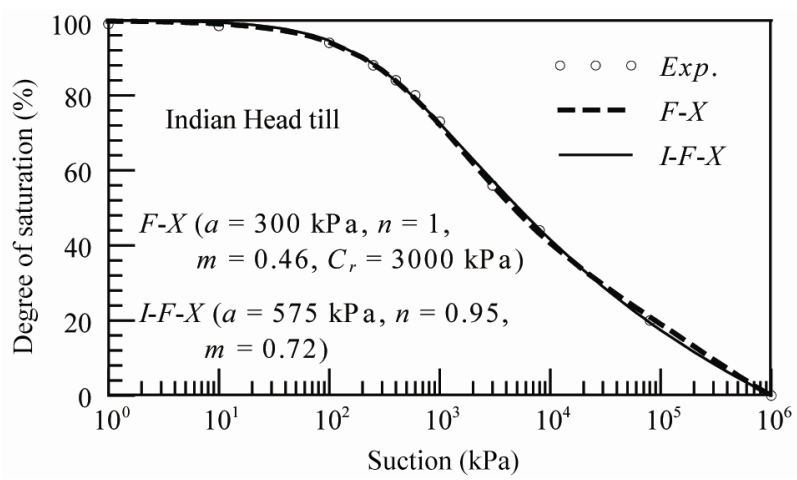

Figure 12. F-X and I-F-X SWCCs for Indian head till.

basically the same but the correction factors $C(\psi)$. The correction factor for the improved model is shown in Equation (16).

$$
K_{r}(\psi)=\frac{\int_{\ln (\psi)}^{b} \frac{\theta\left(e^{y}\right)-\theta(\psi)}{e^{y}} \theta^{\prime}\left(e^{y}\right) \mathrm{d} y}{\int_{\ln \left(\psi_{\text {aev }}\right)}^{b} \frac{\theta\left(e^{y}\right)-\theta_{s}}{e^{y}} \theta^{\prime}\left(e^{y}\right) \mathrm{d} y}
$$

The function $\theta$ is given by the following equation (Equation (15)).

$$
\theta=C(\psi) \frac{\theta_{s}}{\left(\ln \left(e+(\psi / a)^{n}\right)\right)^{m}}
$$

The modified correction factor $C(\psi)$ is given by Equation (16).

$$
C(\psi)=1-\left(\frac{m+1}{m+\left(\psi_{\max } / \psi\right)}\right)^{0.5}
$$

where $\psi_{\max }$ is maximum suction and other parameters are same as in the $F$-All model.

\subsection{Predictive Capability of the Improved Fredlund et al. Model (I-F-All Model)}

The permeability coefficients of water in four different soils are predicted with $F$-All and $I-F$-All models and presented in Figures 13-16. The Figure 13 illustrates the predictions for Superstition sand and the comparison with experimental data (data from [9]). As shown in Figure 13, the $F$-All and $I-F$-All models show better match with the experimental data. However, because of the lack of experimental data, the accuracy of these two models in the higher suction range could not be verified.

The Figure 14 shows the comparison of predicted results and experimental data for Columbia sandy loam (experimental data from [2]). Similar to the Superstition 


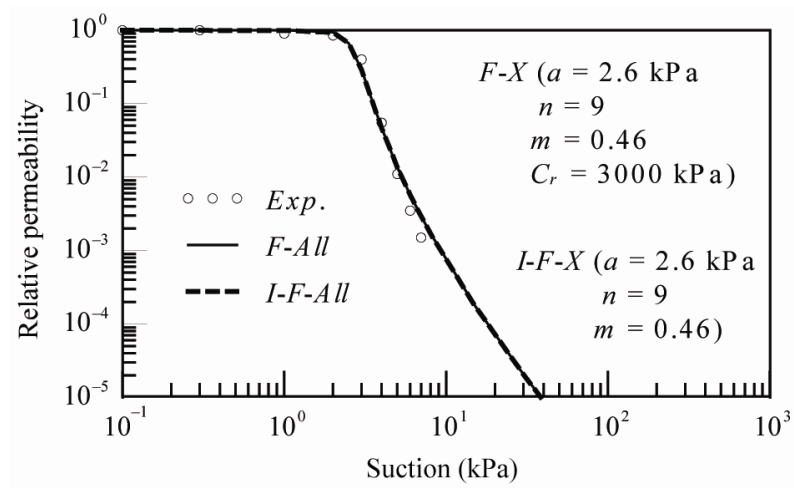

Figure 13. F-All and I-F-All models for Superstition sand.

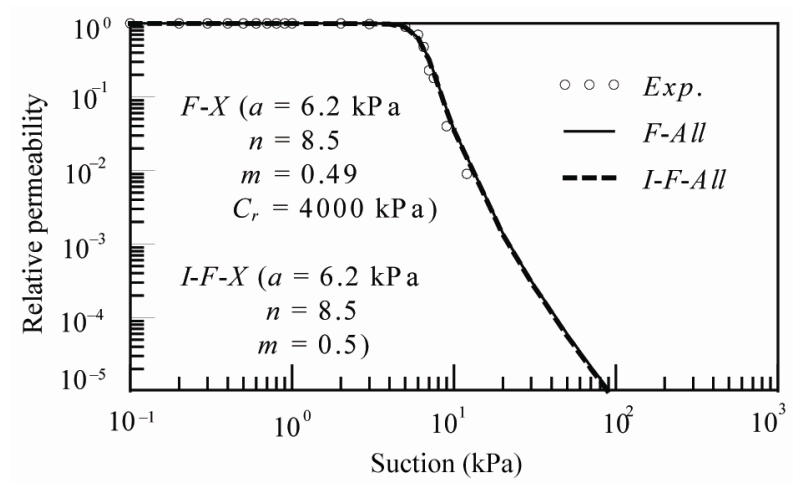

Figure 14. F-All and I-F-All models for Columbia sandy loam.

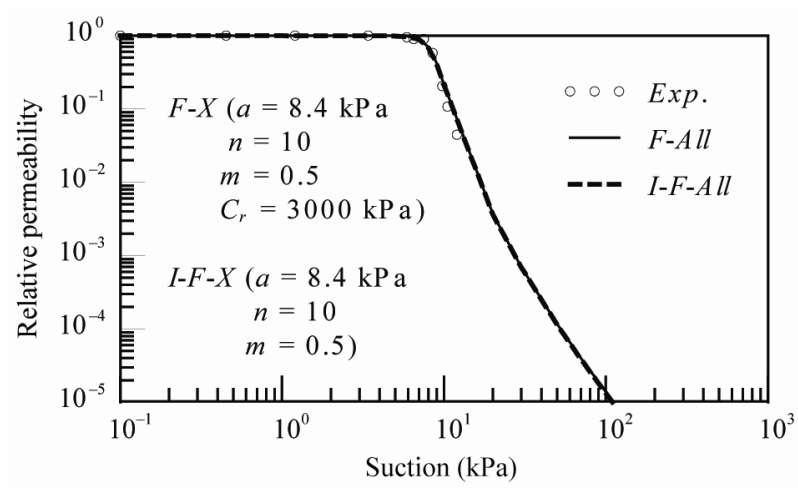

Figure 15. F-All and I-F-All models for Touchet silt loam.

sand, the predictions of $F$-All and $I-F$-All models match well with the experimental data in the lower suction range. As shown in Figure 15, similar predictions are obtained for the Touchet silt loam (experimental data from [2]). The Figure 16 shows the prediction and comparison for Yolo light clay (data from [10]). As shown there, the difference between the experimental data and the predictions of $F$-All and $I-F$-All models increases as the suction increases. In addition, the prediction of $F$-All model slightly deviates from the prediction of $I-F$-All

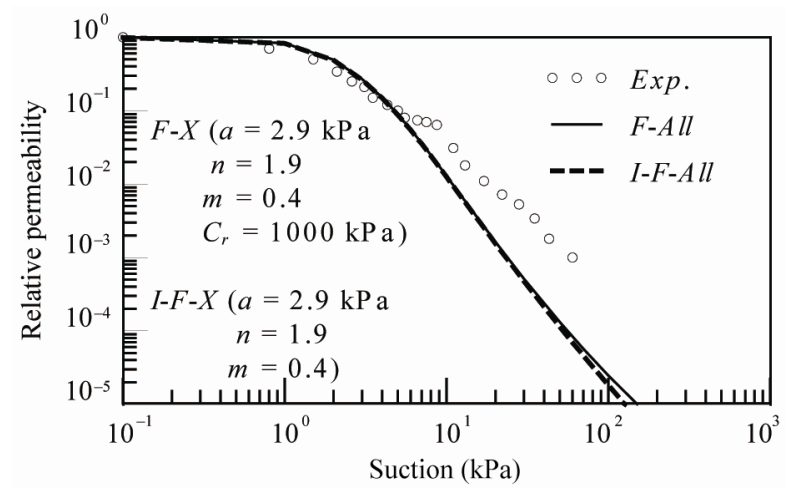

Figure 16. F-All and I-F-All models for Yolo light clay.

model at higher suction range.

\section{Conclusions}

The widely used and most popular soil water characteristic curves (Brooks and Corey, van Genuchten, and Fredlund \& Xing) are modified to capture the high suctions at low degree of saturation. New correction functions are introduced in Brooks-Corey and van Genuchten models and the correction function is the original $F$ - $X$ model was modified as part of this research. The modified equations predict finite suction values at very low degree of saturations. Both maximum suction and residual water content can be used as input in these modified models. The predictive capability of the modified models in low suction range could not be verified because there is no experimental moisture-suction data available at low degree of saturations. However, the flexibility of these modified models has been improved by the introduction of maximum suction as one of the model parameters.

\section{References}

[1] W. Gardner, "Mathematics of Isothermal Water Conduction in Unsaturated Soils,” Highway Research Board Special Report 40, International Symposuim on PhysicoChemical Phenomenon in Soils, Washington DC, 1956, pp. 78-87.

[2] R. H. Brooks and A. T. Corey, "Hydraulic Properties of Porous Media,” Hydrology Paper, Colorado State University, Fort Collins, Vol. 27, No. 3, 1964, pp. 22-27.

[3] M. Th. van Genuchten, “A Closed Form Equation for Predicting the Hydraulic Conductivity of Unsaturated Soils,” Soil Science Society of America Journal, Vol. 44, No. 5, 1980, pp. 892-898. doi:10.2136/sssaj1980.03615995004400050002x

[4] K. Kosugi, "The Parameter Lognormal Distribution Model for Soil Water Retention," Water Resource Research, Vol. 30, No. 4, 1994, pp. 891-901. 


\section{doi:10.1029/93WR02931}

[5] D. G. Fredlund and A. Xing, "Equations for the Soil-Water Characteristic Curve," Canadian Geotechnical Journal, Vol. 31, No. 4, 1994, pp. 521-532.

doi:10.1139/t94-062

[6] D. G. Fredlund, A. Xing and S. Huang, "Predicting the Permeability Function for Unsaturated Soils Using the Soil-Water Characteristic Curve," Canadian Geotechnical Journal, Vol. 31, No. 4, 1994, pp. 533-546. doi:10.1139/t94-062

[7] E. C. Leong, and H. Rahardjo, "Review of Soil-Water Characteristic Curve Equations," Journal of Geotechnical and Geoenvironmental Engineering, Vol. 123, No. 12,
1997, pp. 1106-1117.

doi:10.1061/(ASCE)1090-0241(1997)123:12(1106)

[8] S. K. Vanapalli, D. E. Pufahl and D. G. Fredlund, “The Influence of Soil Structure and Stress History on the Soil-Water Characteristic of a Compacted Till," Geotechnique, Vol. 49, No. 2, 1999, pp. 143-159. doi:10.1680/geot.1999.49.2.143

[9] L. A. Richards, "Water Conducting and Retaining Properties of Soils in Relation to Irrigation," Proceedings of International Symposium on Desert Research, Jerusalem, 1952, pp. 523-546.

[10] R. E. Moore, "Water Conduction from Shallow Water Tables,” Hilgardia, Vol. 1, 1939, pp. 383-426. 